\title{
Elementos estratégicos de la gestión del conocimiento organizacional para la innovación. Caso: red de agrometeorología
}

\author{
Strategic Elements of Organizational Knowledge Management for Innovation \\ Case: Agrometeorology Network \\ Elementos estratégicos da gestão do conhecimento organizacional para a \\ inovação. Caso: rede de agrometeorologia
}

Barlin Orlando Olivares*

Universidad de Córdoba (UCO), Córdoba, España.

Adriana Cortez**

Laboratorio de sistemas de Información en Recursos Agroecológicos, Aragua, Venezuela.

Aura Carolina Muñetones***, Sheyla Casana Barrera****

Universidad Internacional de Andalucía (UNIA), Huelva, España.

RESUMEN. Esta experiencia docente tiene como objetivo proponer un modelo de gestión del conocimiento organizacional para el programa de capacitación desarrollado por el departamento de agrometeorología del Instituto Nacional de Investigaciones Agrícolas (INIA) del estado Anzoátegui, Venezuela. Se utilizó el protocolo de solución de problemas, derivado de la aplicación de ciclos de aprendizaje, denominado TADIR (Traducción, Análisis, Diseño, Implementación y Revisión). Se estableció

Palabras clave: educación, gestión del conocimiento, innovación, aprendizaje. la diversificación de las formas de promover las actividades, tareas, resultados, productos generados por el proyecto, antes, durante y posterior a su ejecución, de manera fácil y completa, así como la conciencia en la población sobre las bondades de la agrometeorológica y su importancia para el desarrollo agrícola del país. Los resultados de esta investigación pueden estar al servicio de la misma organización de manera directa e indirecta y de otras que quieran asumir el reto de contribuir al proceso de transformación que vive la nación. 
ABSTRACT. This teaching experience seeks to propose an organizational knowledge management model for the training program developed by the Agrometeorology Department of the National Institute for Agricultural Research (INAI, in Spanish) of the State of Anzoátegui, Venezuela. The problem solution protocol deriving from the application of learning cycles, TADIR (Translation, Analysis, Design, Implementation and Revision), was used. An array of different ways to promote activities, tasks, results, and products generated by the project was established before, during and after the execution in an easy and complete manner. The population was also made aware of the advantages of agrometeorology and its importance for the agricultural development of the country. The results of this research can be made directly or indirectly available to the same organization or any others who wish to take the challenge of contributing to the transformation process the country is undergoing.

RESUMO. Esta experiência docente tem como objetivo propor um modelo de gestão do conhecimento organizacional para o programa de capacitação desenvolvido pelo departamento de agrometeorologia do Instituto Nacional de Investigações Agrícolas (INIA) do estado de Anzoátegui, Venezuela. Utilizou-se o protocolo de solução de problemas, derivado da aplicação de ciclos de aprendizagem, denominado TADIR (Tradução, Análise, Desenho, Implementação e Revisão). Estabeleceu-se a diversificação das formas de promover as atividades, tarefas, resultados, produtos gerados pelo projeto, antes, durante e após a sua execução, de maneira fácil e completa, assim como sensibilizar a população sobre os benefícios da agrometeorologia e sua importância para o desenvolvimento agrícola do país. Os resultados desta pesquisa podem ser usados pela própria organização de maneira direta e indireta e por outras que queiram assumir o desafio de contribuir ao processo de transformação que vive o país.

Es a partir del siglo XXI, básicamente, cuando se genera un desarrollo considerable en el área de la tecnología, procesos de globalización y sistemas de información en general, que no solo se reflejan en la evolución de las sociedades, sino también en los esquemas organizacionales, representando una oportunidad para contribuir a la renovación de ideas que permitan desarrollar un nuevo tipo de organización acorde con los problemas de la actualidad (Becerra \& Gallego, 2010; Oliveros, 2012).

Justamente, cuando se establece el término de gestión del conocimiento, este se asocia al concepto aplicado en las organizaciones, que busca en principio transferir el conocimiento y la experiencia existente entre sus miembros, de modo que pueda ser utilizado como
Keywords: education, knowledge management, innovation, learning.
Palavras chave: educação, gestão do conhecimento, inovação, aprendizagem. un recurso disponible para otros en la organización (Correa, Jiménez \& Segura, 2008).

En este aspecto, la gestión del conocimiento implica en un sentido muy amplio las técnicas para capturar, organizar, almacenar el conocimiento de los trabajadores, y así transformarlo en un activo intelectual que preste beneficios y se pueda compartir (Canals, 2003; Ongallo, 2003). Por lo tanto, esta gestión aplicada en organizaciones relacionadas con la formación y difusión de información se puede describir como el proceso sistemático de detectar, seleccionar, organizar, presentar y usar la información, para optimizar el cumplimiento de sus objetivos y funciones, logrando que sea sostenible en el tiempo (Albornoz \& Kreimer, 1990; Oliveros, 2012). 
Hoy en día, el conocimiento es el gran tema en todos los ámbitos, sean académicos, científicos, sociales, culturales, empresariales, y en especial, en aquellos que tienen que ver con las ciencias de la información, la inmensa cantidad de información que se dispone sobre cualquier aspecto que le pueda interesar al hombre y su creciente accesibilidad, al disponer de herramientas tecnológicas poderosas que la conectan, se convierte en reflexión obligada para los diferentes agentes que se ocupan de la problemática del conocimiento.

Es precisamente por esta razón que el conocimiento no debe entenderse únicamente como el saber generado por las investigaciones científicas, sino que se deben incluir todas las elaboraciones humanas que pueden ser conceptuales o materiales, que pretendan el desarrollo humano alrededor de las experiencias que conducen al descubrimiento y dominio del mundo para el bien de la humanidad.

Diversos aportes en esta área indican que no es suficiente con tener acceso a grandes cantidades de información documental; es necesario, además, que los individuos puedan y sepan procesar dicha información (Becerra \& Gallego, 2010; Pérez, 2002; Rodríguez, Araujo \& Urrutia, 2001). De nada sirve a cualquier organización, sea una universidad, departamento de investigación, institutos o cualquier otra, poseer individuos con talento y conocimiento si éste no es aplicado en las organizaciones.

La Figura 1 presenta los aspectos más relevantes en el proceso de aprendizaje organizacional; este proceso de la Administración del Conocimiento, también conocido en sus fases de desarrollo como "aprendizaje corporativo" o "aprendizaje organizacional", tiene principalmente tres objetivos que son fundamentales, el primero de ellos está relacionado con identificar, recoger y organizar el conocimiento existente; en segundo lugar, facilitar la creación de nuevo conocimiento y por último, consolidar la innovación por medio de la reutilización y apoyo de la habilidad de la gente a través de organizaciones para lograr un mejor desempeño en la empresa (Davenport \& Prusak, 2001; Martin Fernández, 2001).

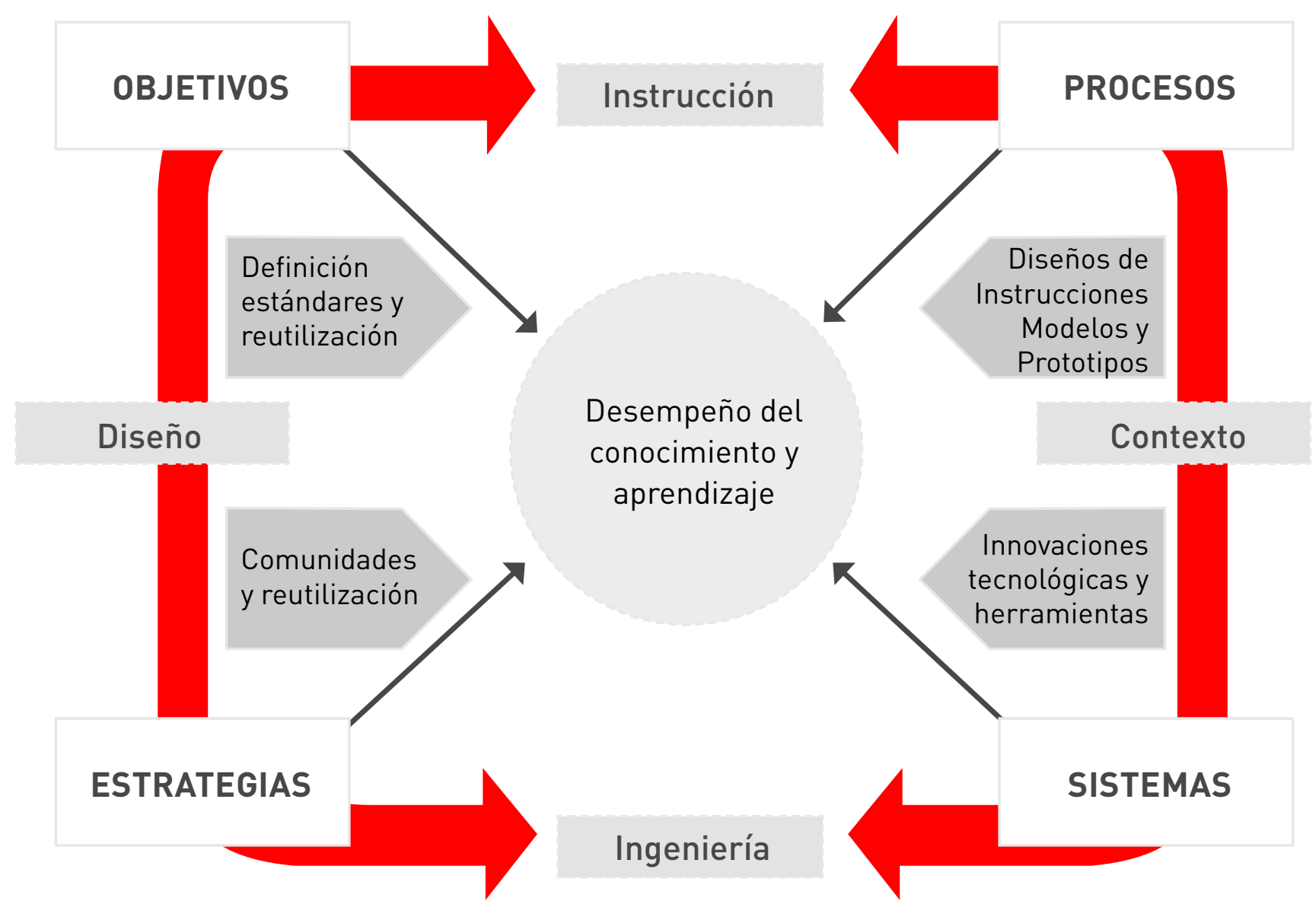

Figura 1. Aspectos relevantes e influyentes en el aprendizaje organizacional. Adaptado de Gestión de Instituciones Educativas Inteligentes. Manual para gestionar cualquier tipo de organización (p. 34), por E. Martin Fernández, 2001, Madrid: McGraw-Hill. Copyright 2001 por McGraw-Hill. 
La transferencia del conocimiento (un aspecto da la Administración del Conocimientol ha existido siempre como proceso en las organizaciones. De manera informal por medio de las discusiones, sesiones, reuniones de reflexión, entre otros y de manera formal por medio del aprendizaje, el entrenamiento profesional y los programas de capacitación.

En la versión de Nonaka y Takeuchi (1995), la gestión del conocimiento cobra nuevas dimensiones en cuanto se trata de lograr que la empresa se convierta en un agente creador de conocimientos y de aprendizajes. Estos autores producen asimismo un salto teórico en cuanto conciben la gestión del conocimiento como parte de una teoría epistemológica con consecuencias filosóficas y sociológicas.

En este orden de ideas, el concepto de la gestión del conocimiento se ha ido enriqueciendo y transformando rápidamente en los últimos años. Si en un primer momento apareció ligado a las teorías de la administración empresaria poco a poco se fue revelando como una idea más compleja ligada a la emergencia de la sociedad del conocimiento y de la información. Al mismo tiempo fue tomando un cariz epistemológico que planteaba la necesidad de repensar un nuevo modo de producción y difusión del conocimiento en las sociedades actuales (Rodríguez et al., 2001). Por otro lado, comenzó a resultar evidente que la gestión educativa estaba íntimamente ligada a la gestión del conocimiento.

Podemos entonces definir provisoriamente la "gestión del conocimiento" como un enfoque epistemológico, organizacional y gerencial, que tiene como fin valorizar y aprovechar la creación y la transmisión de conocimientos en cualquier tipo de organización lempresas,escuelas, hospitales, sindicatos, universidades, cooperativas u organizaciones sin fines de lucrol. Generalmente los documentos de las organizaciones humanas en donde interviene información, conocimientos y consecuentemente aprendizajes, contienen evidencias de lo nuevo y referencias de lo ya conocido (publicado), que deben ser accesibles, útiles y comprensibles para los usuarios, en contextos que comprenden multiplicidad de temas, lugares, tiempos y capacidades. Según Nieminen (2001), esto hace posible el almacenamiento del conocimiento (Knowledge Storage).
Bajo los lineamientos del Proyecto Nacional Simón Bolívar (2007-2013) se establecen las bases firmes para proporcionar a los ciudadanos y ciudadanas de la nación, todos los conocimientos, técnicas y estrategias orientadas al área de agrometeorología, rescate de saberes y planificación agrícola, para su aplicación en el contexto de la comunidad. De esta manera se pretende fortalecer el nivel de organización, con miras a su capacitación para facilitar el aprendizaje obtenido con otros miembros de la comunidad y por ende enriquecer ideológicamente al pueblo, manteniendo la gobernabilidad dentro del mismo.

Interesados en el intercambio de múltiples lecturas sobre el cambio evolutivo de los paradigmas científicos hacia la materialización de una mayor calidad de vida, el servicio de agrometeorología realiza las actividades de investigación, formación y difusión con el propósito de hacer más visible la contribución al desarrollo integral de la sociedad a través de estas tres claves de acción colectivas, como resultados tangibles alcanzados en conjunto con las comunidades del Sur de Anzoátegui, generando respuestas cada vez más contundentes a los problemas agrícolas y aportando mayores opciones en materia de formación en concordancia con los requerimientos de la realidad social de la región.

Ante estas circunstancias, el propósito de esta experiencia es generar reflexiones para considerar el departamento de agrometeorología, como un espacio potencial, no fácilmente reemplazable, para abordar los retos asociados con la innovación tecnológica en las formas de generación y transmisión del conocimiento, capaces de reinventar nuevos paisajes virtuales en coexistencia con la estructura social, científica y académica tradicional que ofrecen posibilidades cognitivas, tanto individuales como grupales.

\section{DESCRIPCIÓN DEL DEPARTAMENTO DE AGROMETEOROLOGÍA}

El objetivo del departamento es generar, procesar y difundir la información agroclimática de la estación climatológica ubicada en El Tigre estado Anzoátegui, con la finalidad de hacerla disponible a los usuarios, dentro de los cuales destacan estudiantes de pregrado y posgrado de diferentes universidades, tecnológicos 
y programas nacionales de formación en las áreas de agroalimentación, gestión ambiental, gestión social y educación integral, investigadores, técnicos, instituciones públicas y privadas, productores agropecuarios, profesionales de diferentes áreas vinculadas con trabajos ambientales (planificadores ambientales, ingenieros, arquitectos, docentes) y público en general. Dentro de los servicios que ofrece están:

a) Datos climáticos (precipitación, evaporación, horarios, diarios, mensuales y anuales)

b. Análisis exploratorio de datos (AED) por medio del programa estadístico Infostat (media, varianza, desviación estándar, coeficiente de variación, curtosis, asimetría, valores máximos y mínimos, percentiles y cuartiles) y la presencia de valores atípicos.

c. Información climática para la planificación de proyectos de investigación y desarrollo agrícola, apoyo de seguros agrícolas, diseño de obras de saneamiento, sistema de alerta y prevención de desastres.

d. Visitas guiadas a la estación climatológica con fines didácticos, haciendo énfasis en cada uno de los instrumentos meteorológicos que se encuentran en esta estación. e. Asesorías vinculadas con caracterizaciones agroclimáticas y ambientales de la región oriental.

f. Bibliografía especializada (Publicaciones, boletines climatológicos, libros entre otros).

g. Cursos y eventos de formación y capacitación en el área de agrometeorología y su relación con otras áreas como manejo de suelos, manejo agroecológico de cultivos, evaluación de pastos y forrajes, sanidad y manejo animal y sistemas de producción agrícola.

\section{DESCRIPCIÓN DEL MODELO CONCEPTUAL DE GESTIÓN DEL CONOCIMIENTO ORGANIZACIONAL}

Para dar inicio a la experiencia, se estableció la conceptualización de la gestión estratégica del conocimiento la cual es considerada de reciente data (1995), y su origen responde a un proceso que se inicia con el tema de la Gestión por Competencias y el desarrollo de las TIC para crear ventajas competitivas en economías que tienden a centrarse en el conocimiento y el aprendizaje (Peluffo \& Catalán, 2002; Romero, 2005). Partiendo de este punto, la figura 2 representa las tres principales perspectivas consideradas en el estudio, así como los focos de atención.
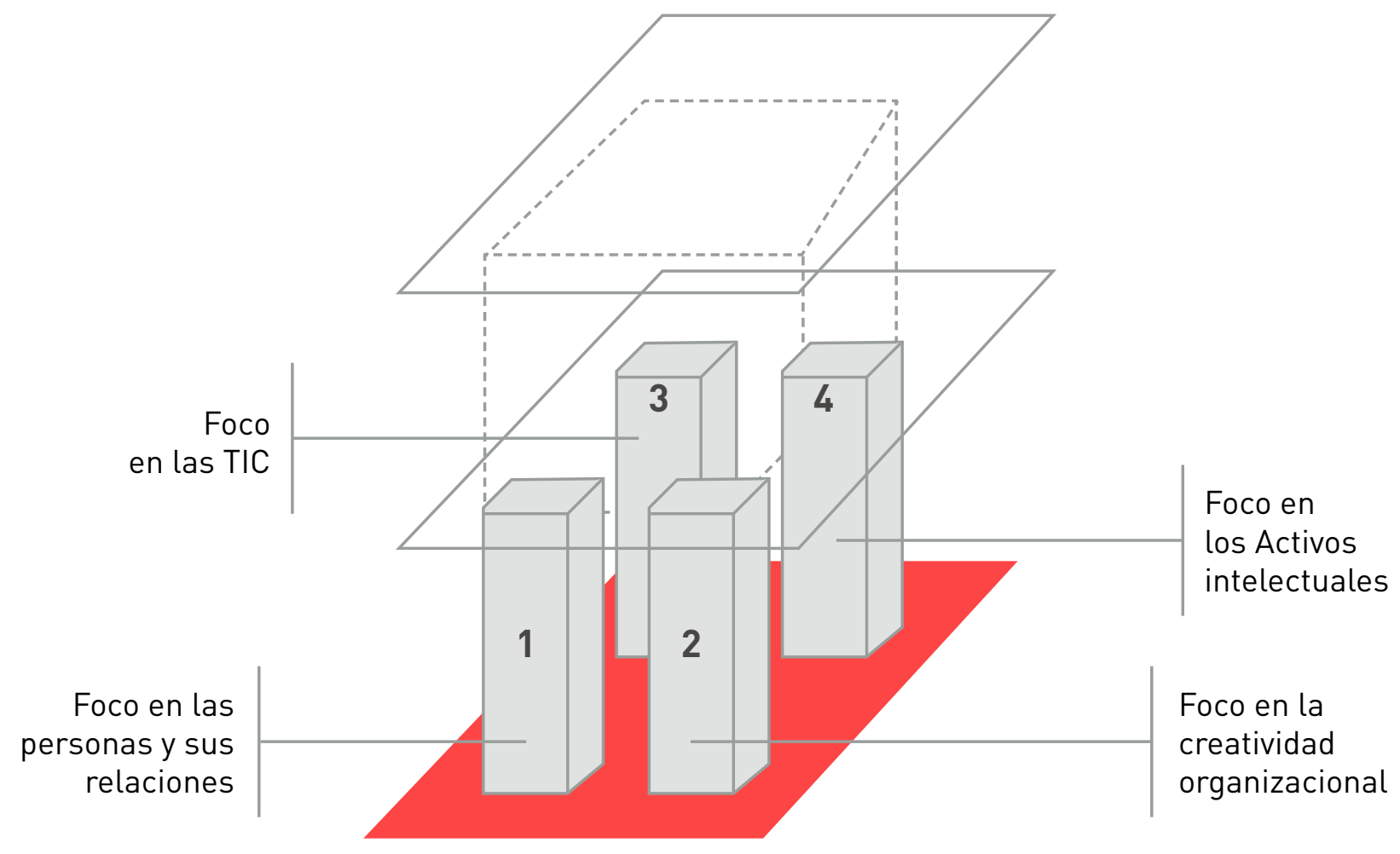

Perspectiva estratégica creatividad organizacional

Perspectiva operacional

Figura 2. Áreas focales para una estrategia de Gestión de Conocimiento. Adaptado de Introducción a la gestión del conocimiento y su aplicación al sector público (p. 12), por A. M. B. Peluffo y C. E. Catalán, 2002, Santiago de Chile: Instituto Latinoamericano y del Caribe de Planificación Eco-nómica y Social - ILPES. Copyright 2002 por Naciones Unidas. 
La obtención de soluciones a problemas en sistemas de aprendizaje humano requiere de la construcción de modelos conceptuales con tres ingredientes: un marco teórico, un escenario logístico y un protocolo de solución que los integra y hace funcionar (Barojas, 2002).

En primer lugar, cabe mencionar que el marco teórico permite entender las creencias, ideales, conceptos, actitudes y valores de los miembros de la comunidad de aprendizaje involucrados en la solución del problema y del contexto en el cual este se define. En segundo lugar, el escenario logístico lo conforman las condiciones y los principios de operación de dicha comunidad, y se refiere tanto a los recursos humanos, materiales y tecnológicos con los que cuenta, como a las habilidades prácticas que hacen posible su funcionamiento. En este sentido, el protocolo de solución se refiere a los procedimientos considerados pertinentes para dar respuesta a los problemas de interés en la comunidad de aprendizaje.

Barojas y Dehesa (2001) y Duval (1993) proponen un protocolo de solución de problemas derivado de la aplicación de ciclos de aprendizaje en donde intervienen lenguajes naturales, técnicos y formales, así como diversos registros de representación semiótica, tales como textos, símbolos, fórmulas, modelos, esquemas, curvas, gráficas, tablas y códigos.

Este protocolo se denomina TADIR, en referencia explícita a las iníciales de las cinco etapas que lo componen (Barojas \& Pérez, 2001): T-Traducción, A-Análisis, D-Diseño, I-Implementación y R-Revisión. Las primeras cuatro etapas (TADI) definen la dimensión cognitiva del protocolo y sirven en la construcción del modelo conceptual requerido para obtener la solución, mientras que la quinta (R) se refiere a la dimensión metacognitiva y ayuda en la evaluación de la solución obtenida (ver Tabla 1).

Ahora bien, según Nonaka y Takeuchi (1995), en la creación y gestión del conocimiento conviene considerar que este es de dos tipos: el tácito (T) y el explícito (E), por lo que pueden establecerse cuatro posibles modos de conversión entre los tipos Tácito y Explícito. De acuerdo con lo anterior, la creación del conocimiento organizacional pasa por las siguientes cinco fases: en primer lugar, compartir conocimiento tácito (socialización: del Tácito al Tácito), segundo, creación de conceptos (externalización: del Tácito al Explícito), tercer lugar, justificación de conceptos (internalización: del Explícito al Tácito), cuarto, construir arquetipos o modelos y por último, obtener conocimiento cruzado (combinación: de Explícito a Explícito).

Para este estudio se describe la aplicación del protocolo TADIR en la formación de estudiantes de pregrado en el área de agrometeorología, actividad perteneciente al subproyecto: Desarrollo y trasferencia de tecnología e información con base en investigación agrometeorológica, apoyada en el fortalecimiento y ampliación de la red agrometeorológica del estado Anzoátegui.

La figura 3 muestra el modelo combinado de aprendizaje organizacional, el cual sirvió de base para el estudio. Haciendo hincapié en la perspectiva del cambio abordada en el estudio de una serie de contenidos teóricos que implican algún tipo de relación entre el Aprendizaje Organizativo y el cambio organizativo.

\section{APLICACIÓN DEL MODELO DE GESTIÓN DEL CONOCIMIENTO ORGANIZACIONAL EN EL DEPARTAMENTO DE AGROMETEOROLOGÍA}

Ahora bien, para orientar la administración del conocimiento organizacional se concretaron cuatro metas y los criterios para determinar que cada una de las metas fuera alcanzada, fortaleciendo los procesos de formación o capacitación, según se observa en la tabla 2.

Para determinar el cumplimiento de las metas anteriormente indicadas se establecieron dos criterios: el primero está relacionado con el ámbito cualitativo, al observar la puesta en práctica de los mecanismos de gestión del conocimiento; y el segundo criterio relacionado con el ámbito cuantitativo cuando implica algún tipo de producción, como reportes, tesis y artículos. Luego se presenta un resumen de la solución propuesta al aplicar el protocolo TADIR a la población de estudiantes que participó en los eventos desarrollados por el servicio. 
Tabla 1

Descripción del protocolo TADIR y su aplicación a problemas en sistemas de aprendizaje hu-mano (Barojas, 2003)

ETAPA

\begin{tabular}{|l|l}
\hline & $\begin{array}{l}\text { El enunciado del problema suele estar } \\
\text { redactado en lenguaje cotidiano (natural), } \\
\text { por lo que se reformula en el lenguaje de } \\
\text { Ta disciplina correspondiente. }\end{array}$
\end{tabular}

Diseño

Implementación

Análisis requeridas para interpretar y construir la solución del problema, tomando en cuenta los modelos y teorías que sean relevantes.

Se propone un esquema o diagrama conceptual que muestra la línea de razonamiento que incluye los conceptos, argumentos, evidencias y demostraciones por utilizar en la solución del problema.

Se lleva a la práctica el camino señalado en el Diseño y se incluyen las definiciones, criterios y demás elementos informativos y procedimentales

\section{APLICACIÓN}

Busca dar respuesta a: ¿quiénes integran la comunidad de aprendizaje que tiene a su cargo la solución del problema?, ¿en qué actividades de transformación están involucrados?. ¿con qué propósitos?, ¿en qué temática? y ¿con qué recursos?

Busca describir los principales factores que explican el funcionamiento del sistema y señalar cuáles son sus objetivos, restricciones y condiciones de conectividad

Propone una representación gráfica de los elementos del espacio cognitivo de la solución referidos a tres actividades de transformación: (1) investigación educativa con propósitos de comprender los factores críticos y los principios operacionales, (2) desarrollo para hacer que las cosas funcionen con eficiencia de acuerdo con lo previsto, con calidad y éxito, y (3) comunicación asociada con la emisión, recepción e interpretación de mensajes. necesarios para resolver el problema, usando lenguajes natural, técnico o formal.

Utilización de mecanismos de monitoreo y control para poner en práctica la solución del problema. El mecanismo de monitoreo se define en términos de las denominadas "habilidades del siglo XXI": alfabetización digital, pensamiento inventivo, comunicación efectiva y alta productividad (NCREL, 2000).

Se consideran todas y cada una de las etapas anteriores para detectar posibles errores conceptuales, suposiciones falsas o erróneas, cálculos equivocados, resultados obtenidos en condiciones que muestren ser inaplicables o inadecuadas, así como comparar la respuesta obtenida con la prevista, replantear el problema,
Cada una de las actividades de transformación es considerada como un subsistema para el cual se define su significado y se especifican los correspondientes procesos o productos. 


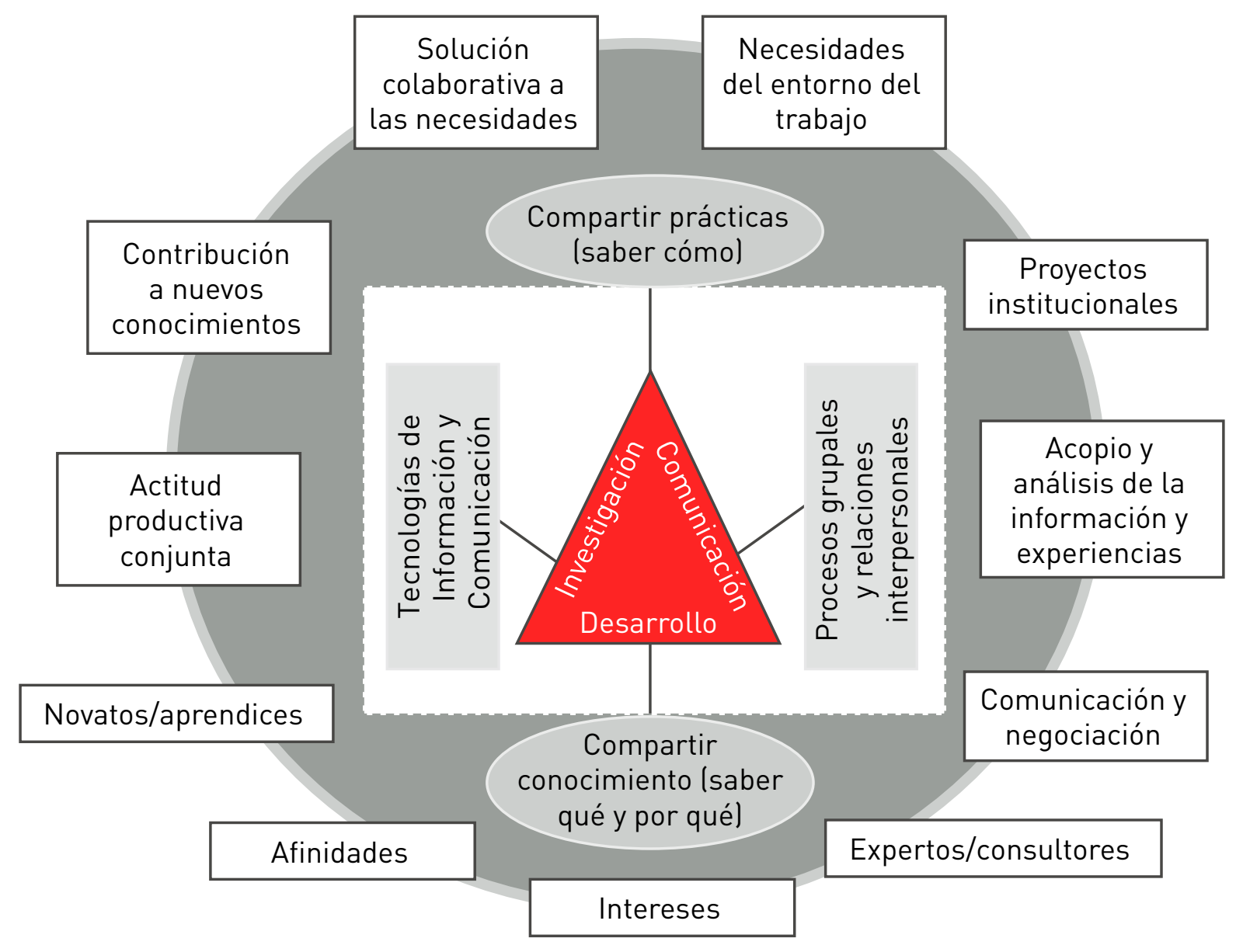

Figura 3. Modelo combinado de Aprendizaje Organizacional. Adaptado de "The Learning Organization: Change Proofing and Strategy”, por S. Drew \& P. Smith, 1995, The Learning Organization, 2(l) (p. 10), Copyright 1995 por MCB University Press.

Tabla 2

Descripción de las metas y criterios establecidos

\begin{tabular}{|c|c|c|}
\hline META & DENOMINACIÓN & DESCRIPCIÓN \\
\hline 1 & Abordaje & $\begin{array}{l}\text { Construcción, implementación y análisis del uso de instrumentos } \\
\text { para identificar los recursos, necesidades, amenazas, fortalezas y } \\
\text { posibilidades de los usuarios del servicio }\end{array}$ \\
\hline 2 & Formación & $\begin{array}{l}\text { Eventos tales como cursos, talleres, coloquios y jornadas presenciales } \\
\text { y a distancia para promover el conocimiento en agrometeorología } \\
\text { con la integración de recursos multimedia, así como el desarrollo de } \\
\text { presentaciones, de material didáctico y de contribuciones al portal } \\
\text { electrónico de la red }\end{array}$ \\
\hline 3 & Evaluación & $\begin{array}{l}\text { Consideración de evidencias que muestren mejoría en el desempeño de } \\
\text { los usuarios }\end{array}$ \\
\hline 4 & Implementación & $\begin{array}{l}\text { Comparación de los logros alcanzados en los usuarios bajo estudio y } \\
\text { proposición de otras aplicaciones y extensiones }\end{array}$ \\
\hline
\end{tabular}


Fase de Traducción. En el contexto de la sociedad actual, caracterizada por el uso de la información y el conocimiento, la educación constituye un factor imprescindible. En primer lugar, por razones de equidad social, para que se cumpla la responsabilidad del estado venezolano de proporcionar a todos los ciudadanos (as) iguales capacidades que les permitan aprovechar las oportunidades; y en segundo lugar, porque los recursos invertidos en programas de formación y educación son la mejor manera de asegurarle a un país, a mediano y largo plazo, mayor crecimiento y equidad, mejorando las habilidades y destrezas que pueden utilizarse en el trascurso de la vida y tienen efectos directos sobre los ingresos futuros.

La finalidad de esta fase de traducción fue conformar una comunidad de aprendizaje en la que los estudiantes de los diferentes institutos y universidades del Sur de Anzoátegui puedan explorar las habilidades y potencialidades de cada uno, haciendo énfasis en que cada uno pueda aprender. Así mismo se pretende que los miembros de esta comunidad de aprendizaje puedan compartir sus experiencias e intercambien comunicaciones con apoyo de recursos orientados hacia las tecnologías de información y comunicación, de tal manera que conjuntamente se vaya construyendo un conocimiento organizacional.

Fase de Análisis. Cada uno de los eventos de formación en el área agrometeorológica estableció el perfeccionamiento de las habilidades de los estudiantes, la adquisición de nuevas competencias y el desarrollo de las aptitudes para desempeñarse en la profesión u oficio relacionado con el agro. En este sentido, los cursos de formación estuvieron orientados hacia la enseñanza centrada en los fundamentos de los saberes y no simplemente en los procedimientos, como ha sido tradicionalmente en las universidades venezolanas.

La solución del problema se establece dentro de un modelo constructivista para la enseñanza de las ciencias naturales. Es conveniente indicar que dentro de las limitaciones más frecuentes que se encuentran en las instituciones y universidades que imparten carreras relacionadas con las ciencias del agro del Sur de Anzoátegui están el alto porcentaje de profesores y facilitadores con edad avanzada, lo que genera cierto temor en el empleo de las tecnologías de información y comunicación; también, la poca disponibilidad de recursos económicos y técnicos de los estudiantes; y por último, el desarrollo estricto del modelo de enseñanza tradicional a el que están sujetos los estudiantes.

Es destacable que la mayoría de los estudiantes participantes estuvieron muy dispuestos para insertarse en los eventos donde se aborde el tema agrometeorológico con la tecnología. De esta manera se genera la posibilidad al estudiante de apropiarse del proceso formativo y la adquisición de una visión más integral de su profesión, así como mirar los planes de estudio desde la óptica social, política y agrícola, con el propósito de mejorar el uso racional de los recursos y posibilitar un compromiso mayor con el desarrollo del conocimiento.

Fase de Diseño. En esta fase se elaboró una representación gráfica (ver Figura 4) de los elementos del espacio cognitivo de la solución referidos a tres actividades de transformación: (1) investigación educativa con propósitos de comprender los factores críticos y los principios operacionales, (2) desarrollo para hacer que las cosas funcionen con eficiencia de acuerdo con lo previsto, con calidad y éxito, y (3) comunicación asociada con la emisión, recepción e interpretación de mensajes.

Fase de Implementación. Durante el periodo 20092013 se desarrollaron una serie de cursos, talleres, conversatorios dirigidos fundamentalmente a estudiantes, docentes y productores de comunidades agrícolas (ver Tabla 3), estos eventos fueron en conjunto con: la Facultad de Agronomía de la Universidad Nacional Experimental Politécnica de la Fuerza Armada (UNEFA), La Universidad Bolivariana de Venezuela (UBV), Misión Sucre, P.N.F. en Gestión Ambiental, El Instituto Universitario de Tecnología José Antonio Anzoátegui (IUTJAA) sede El Tigre y Pariaguán. Los eventos tuvieron como objetivos, socializar y evaluar las potencialidades, limitaciones y características climáticas de los sistemas ambientales a fin de mejorar la producción y su calidad, reducir las pérdidas y riesgos climáticos, reducir costos, mejorar la eficiencia del uso del agua, trabajo y energía, así como conservar los recursos potenciales de la región.

La figura 5 muestra los diferentes tipos de formación en los cuales participaron estudiantes de las diferentes 


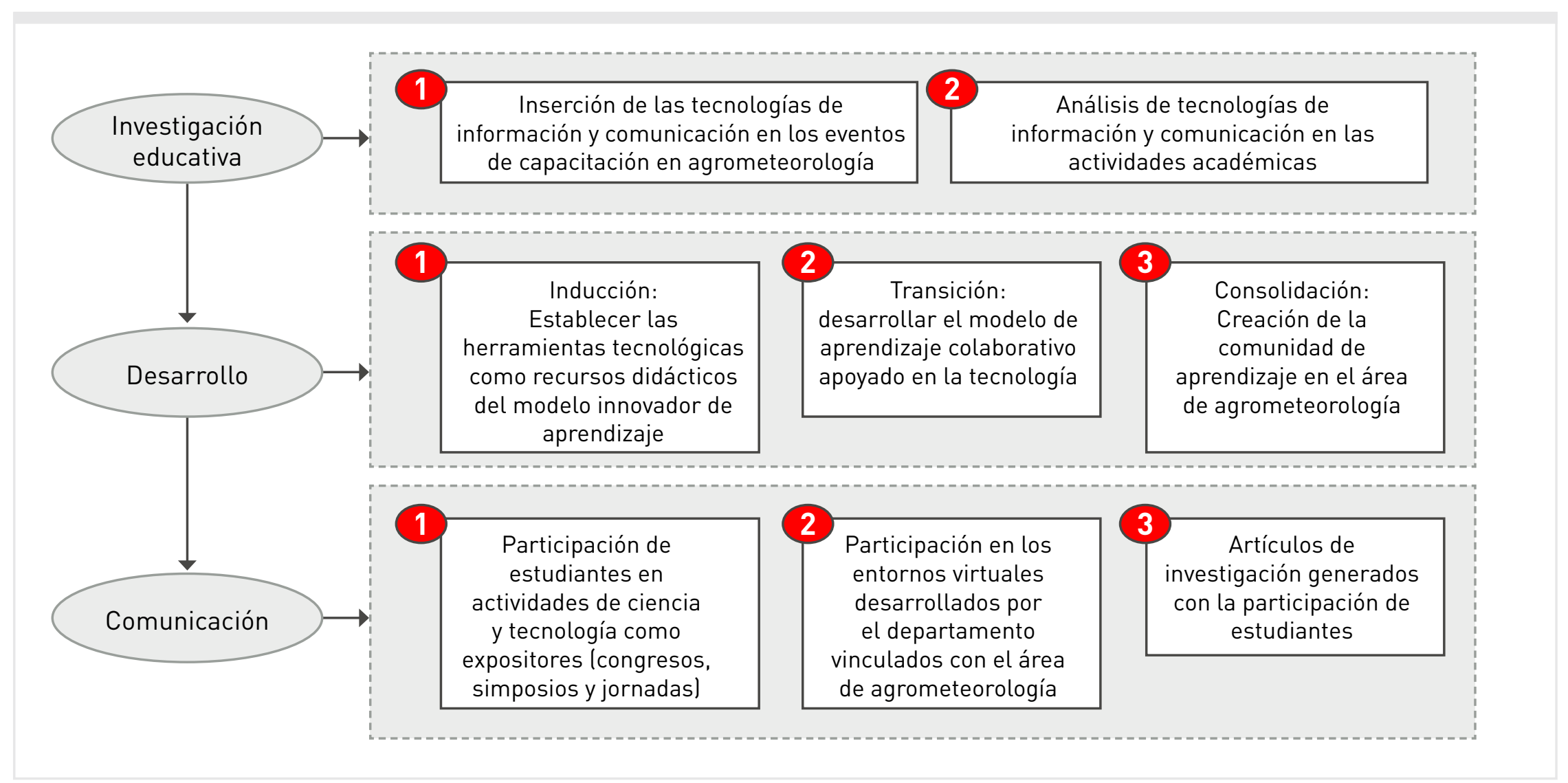

Figura 4. Representación gráfica de los elementos del espacio cognitivo de la solución referida en las tres actividades de transformación. Adaptado de "Mathematics for Social Scientists: Learning Cycles and Teaching Strategies", por J. Barojas \& N. Dehesa, 2001, Industry and Higher Education, 15(4) (p.7), Copyright 2001 por IP Publishing Ltd.

\section{TIPO DE PARTICIPACIÓN}

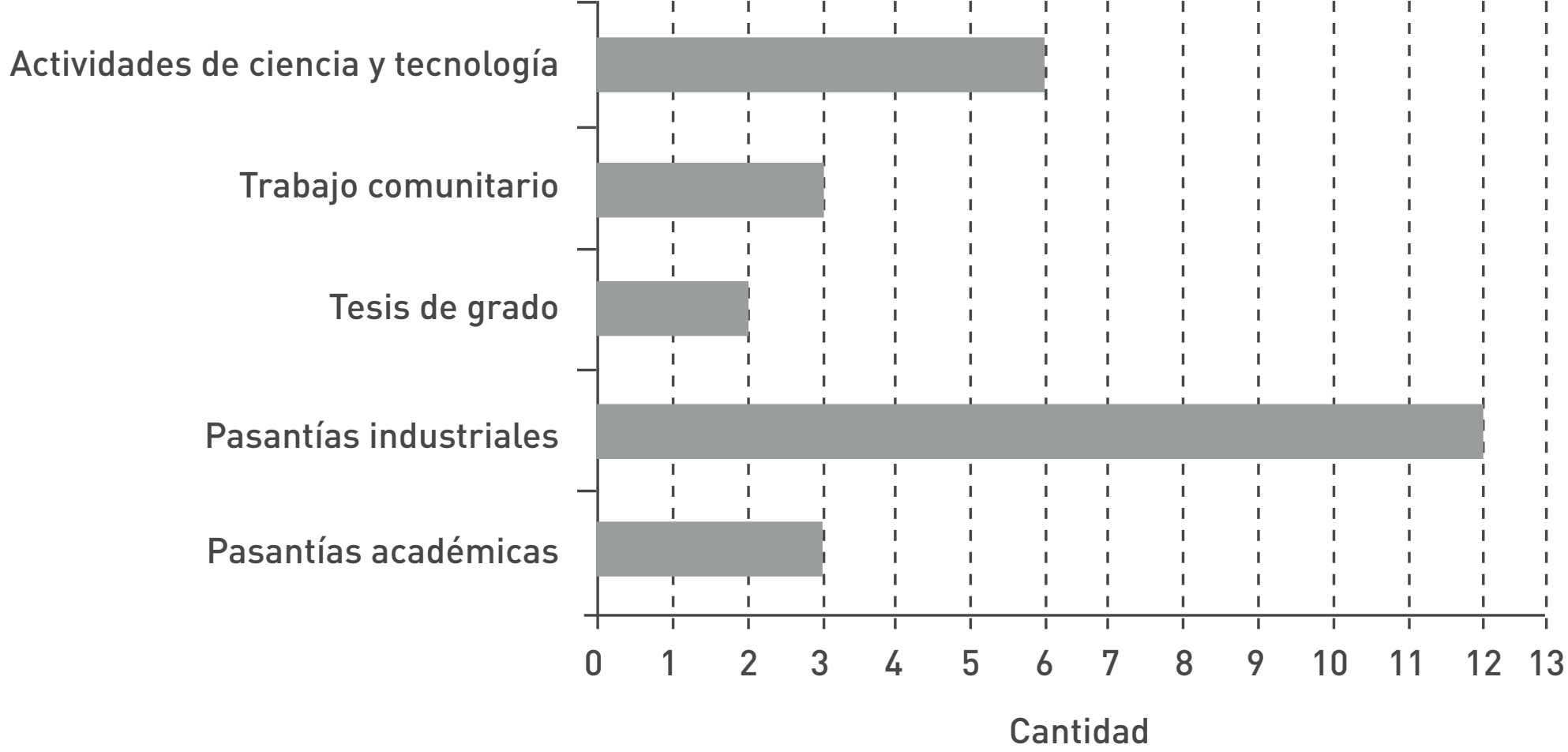

Figura 5. Participación de estudiantes en los diferentes tipos de formación abordados en el proyecto la red agrometeorológica de Anzoátegui de periodo 2009-2013 
Tabla 3

Eventos desarrollados por el Servicio de Agrometeorología del INIA Anzoátegui periodo 2009-2013

\begin{tabular}{|c|c|c|c|}
\hline $\mathrm{N}$ & EVENTO & $\begin{array}{l}\text { INSTITUCIÓN/ } \\
\text { COMUNIDAD }\end{array}$ & PARTICIPANTES \\
\hline 1 & Curso: Determinación del requerimiento hídrico de los cultivos & UNEFA & 27 \\
\hline 2 & $\begin{array}{l}\text { Curso Taller: Alternativas agroecológicas para el manejo } \\
\text { agronómico de cultivos }\end{array}$ & UNEFA y UBV & 25 \\
\hline 3 & $\begin{array}{l}\text { Conversatorio: Intercambio de Experiencias Internacionales: } \\
\text { conectando países para el fortalecimiento del conocimiento } \\
\text { climático local latinoamericano }\end{array}$ & $\begin{array}{l}\text { IUTJAA El Tigre y } \\
\text { Pariaguán }\end{array}$ & 12 \\
\hline 4 & $\begin{array}{l}\text { Curso teórico practico: Interpretación de información climática } \\
\text { con fines agrícolas y ambientales }\end{array}$ & UNEFA & 34 \\
\hline 5 & $\begin{array}{l}\text { Curso Taller: Manejo agroecológico de cultivos en la Mesa de } \\
\text { Guanipa }\end{array}$ & IUTJAA El Tigre & 35 \\
\hline 6 & $\begin{array}{l}\text { Curso Taller: Generalidades sobre el manejo sostenible del suelo } \\
\text { bajo Bosque Seco Tropical }\end{array}$ & IUTJAA Pariaguán & 41 \\
\hline 7 & $\begin{array}{l}\text { Taller práctico: Manejo sostenible del suelo en sistemas de } \\
\text { producción agrícola bajo Bosque Seco Tropical }\end{array}$ & IUTJAA El Tigre & 85 \\
\hline 8 & $\begin{array}{l}\text { Curso Taller: Características de interés agrícola de la } \\
\text { precipitación }\end{array}$ & IUTJAA Pariaguán & 42 \\
\hline 9 & $\begin{array}{l}\text { Curso: Aspectos claves para la toma de datos en la estación } \\
\text { climática }\end{array}$ & UBV- Misión Sucre & 18 \\
\hline 10 & $\begin{array}{l}\text { Taller práctico: Sensibilización acerca del conocimiento } \\
\text { agrometeorológico }\end{array}$ & UNEFA Aguasay & 29 \\
\hline 11 & $\begin{array}{l}\text { Curso: Socialización del conocimiento Agrometeorológico en el } \\
\text { sistema ambiental }\end{array}$ & UNEFA y UBV & 21 \\
\hline 12 & $\begin{array}{l}\text { I Coloquio sobre Manejo Sostenible del Suelo en La Mesa de } \\
\text { Guanipa }\end{array}$ & UNEFA & 21 \\
\hline 13 & $\begin{array}{l}\text { I Jornada participativa sobre reconocimiento y evaluación de } \\
\text { gramíneas forrajeras y leguminosas en condiciones de sabana }\end{array}$ & UNEFA & 12 \\
\hline 14 & $\begin{array}{l}\text { II Coloquio sobre Manejo Sostenible del Suelo en La Mesa de } \\
\text { Guanipa }\end{array}$ & IUTJAA El Tigre & 25 \\
\hline 15 & Taller Caracterización del régimen hídrico con fines agrícolas & IUTJAA El Tigre & 12 \\
\hline 16 & $\begin{array}{l}\text { Taller de construcción popular denominado: manejo } \\
\text { agroecológico y rescate de saberes del cultivo de yuca }\end{array}$ & Kashaama & 16 \\
\hline 17 & $\begin{array}{l}\text { Taller de participación colectiva denominado: fundamentos } \\
\text { agroecológicos para el cultivo de batata en los llanos orientales }\end{array}$ & Kashaama & 12 \\
\hline 18 & $\begin{array}{l}\text { Mesa de trabajo para el fortalecimiento del poder popular } \\
\text { denominada: jerarquización de la información agrosocial como } \\
\text { herramienta para promover la organización comunal }\end{array}$ & Kashaama & 17 \\
\hline
\end{tabular}


instituciones educativas mencionadas anteriormente. En principio, se pretende de forma integral promover las actividades programadas en el marco del proyecto de la red agrometeorológica de Anzoátegui, insertando nuevos patrones de información para la divulgación de temas asociados con la agrometeorología, que de alguna manera garanticen el empoderamiento del conocimiento en territorios agrícolas rurales.

Este proyecto puso a disposición distintos medios o recursos audiovisuales y escritos, apoyados en las nuevas tecnologías de información y comunicación para promover, difundir y divulgar las diferentes aplicaciones y productos generados en el ámbito agrometeorológico que estarán al servicio del pueblo y para el pueblo. La Tabla 4 presenta el número de productos de investigación y divulgación que se generaron en el departamento con la participación activa y protagónica de los estudiantes del Sur de Anzoátegui.

También se generaron dos entornos virtuales, que permitieron a los participantes vincularse con recursos que no habían utilizado anteriormente y socializar con profesionales y miembros de sociedades científicas a nivel nacional e internacional. En síntesis, la didáctica tecnológica es una modalidad educativa que surge de la necesidad propia de la educación y tecnología educativa.
Es indispensable que la innovación tecnológica se acompañe de innovación pedagógica, para lo cual es necesario incorporar los cambios estrictamente técnicos en el marco de proyectos educativos y en especial a los docentes. La educación actual debe apoyar proyectos pedagógicos que promuevan la construcción del conocimiento; esto exige planes didácticos concretos apoyados en los soportes tecnológicos que estimulen la reflexión, el análisis, la proposición y la ejecución como procesos para la significatividad del aprendizaje.

Fase de Revisión. El punto de reflexión es precisamente el impacto que ha tenido la tecnología en la educación en los procesos de capacitación, donde se conoce que dicho impacto ha sido nulo, principalmente por la poca intención del sector educativo en adaptarse al nuevo perfil del alumno de esta nueva era digital. En este sentido, se requiere de un cambio profundo en la forma de educar o formar a las personas, se debe trasformar la manera de educar basada en la obediencia y el castigo, orientando justamente el proceso a la vinculación del estudiante con un entorno atractivo ligado al uso de la tecnología.

En este sentido, la solución propuesta ha mostrado que puede generar conocimiento organizacional dentro del programa de formación para estudiantes del sector

\section{Tabla 4}

Productos de investigación y divulgación generados en el proyecto durante el periodo 2009-2013 con la participación de estudiantes del Sur de Anzoátegui

\section{TIPO DE PRODUCTO CANTIDAD}

\begin{tabular}{|l|c|c|}
\hline Artículos científicos & 9 & $\begin{array}{l}\text { Variabilidad climática, control de calidad de datos, percepción local del } \\
\text { clima, fitopatología y análisis espacio temporal de la precipitación }\end{array}$ \\
\hline Capítulos de libros & 2 & Manejo agronómico del cultivo de soya \\
\hline Articulo divulgativo & 12 & $\begin{array}{l}\text { Características de la lluvia, importancia de la agrometeorología, } \\
\text { conocimiento local agrometeorológico, riego y agrometeorología, } \\
\text { sistemas de riego, conservación de suelos y agua }\end{array}$ \\
\hline Total & 23 & \\
\hline
\end{tabular}


universitario del Sur de Anzoátegui. Sin embargo hay ciertas limitaciones, como la falta de tiempo por parte de los estudiantes en participar en estos eventos de formación, también el escaso interés de los profesores 0 instituciones educativas en insertar a los estudiantes en eventos de este tipo. Es conveniente indicar que la mayoría de los estudiantes disponen de adecuados equipos de computación que permitieron la amplia participación en los entornos virtuales.

\section{CONCLUSIONES}

De esta experiencia se concluye que son muchos los aspectos que hay que considerar para lograr una verdadera gestión del conocimiento en las instituciones educativas. En principiose requiere fortalecer el enfoque complejo y estratégico a fin de formular políticas de conocimiento tomando como referencia los aspectos epistemológicos, pedagógicos, organizacionales y sociales de los programas científicos y tecnológicos y académicos de la institución; en segundo lugar consolidar la coherencia institucional definiendo los fines, misión, visión, funciones y objetivos; luego asegurar la congruencia organizacional y por último, lograr la estrecha interrelación entre las funciones básicas de la institución lenseñanza, investigación, extensión y difusión).

A manera de reflexión, para el personal vinculado con el proyecto, la experiencia significó un avance importante en toda la trayectoria de la institución debido fundamentalmente a la implementación de los elementos estratégicos de la gestión del conocimiento organizacional para la innovación. Dentro de los productos de la Red de agrometeorología destacan publicaciones en revistas de amplia difusión en el país, la formación de una cantidad considerable de personas de diferentes ámbitos y por último, la difusión de la ciencia en espacios comunales. Para los alumnos participantes en las actividades vinculadas con la red, esta experiencia representa la base para incentivar mayor incursión en el área de investigación agrícola, docencia o extensión.

Los resultados aquí expuestos pueden ser replicables fácilmente en otros contextos e instituciones de educación superior en cuanto a la implementación de un proceso de gestión del conocimiento como el que se ha aplicado, siempre y cuando se tomen en cuenta los elementos estratégicos promovidos en esa experiencia, el tipo de proyecto por desarrollar y el público involucrado.

Los aportes importantes realizados por Barojas (2002); Barojas y Pérez (2001) y Barojas (2003) establecen como conclusión que los sistemas educativos operan con más eficiencia, siendo más dinámicos y flexibles, siempre y cuando se insista que el capital estructural sirve integralmente solo si existe un capital humano que lo sepa utilizar, implicando una eficiente gestión del conocimiento organizacional. Este conocimiento habrá de desarrollarse en organizaciones humanas que hacen uso extensivo de las tecnologías de la información y comunicación, con el fin de enriquecer los conocimientos y aprendizajes, tanto en los individuos como en las organizaciones.

Debido a los grandes y significativos cambios que genera la transición hacia un nuevo modelo de producción agrícola, se requiere en un sentido muy amplio del desarrollo de diferentes formas de difusión del conocimiento. Pero a la vez la diversificación de las formas de promover las actividades, tareas, resultados, productos generados por el proyecto, antes, durante y posterior a su ejecución, de manera fácil y completa, llegando a la población en momentos oportunos y con gran contenido que impacte y promueva la facilitación de adopción de la tecnología e innovaciones, así como la conciencia en la población de las bondades de la agrometeorología y su importancia para el desarrollo agrícola del país.

En función a lo anterior, se pretende que la utilización efectiva de la gestión del conocimiento permita realizar más eficazmente el trabajo encomendado a la organización. Así mismo, mediante la gestión del conocimiento las organizaciones favorecen a que el individuo se desarrolle en su trabajo aportando ideas, al mismo tiempo que evita la "fuga de conocimiento". 


\section{REFERENCIAS}

Albornoz, M \& Kreimer, P. (1990). Ciencia y Tecnología: Estrategias y políticas de largo plazo. Buenos Aires: Eudeba.

Barojas, J. (2002). Comunidades de Aprendizaje y organización del conocimiento. Ponencia presentada en XVIII Simposio Internacional de Computación en Educación en el tema de comunidades de aprendizaje, SOMECE (Eds.), Zacatecas, México.

Barojas, J. (2003). Teacher training as collaborative problem solving. Educational Technology and Society, 7(1), 21-28. Recuperado de http://www.ifets.info/journals/7_1/4.pdf

Barojas, J., \& Dehesa, N. (2001). Mathematics for Social Scientists: Learning Cycles and Teaching Strategies. Industry and Higher Education, 15(4), 269-277. doi: 10.5367/000000001101295768

Barojas, J., \& Pérez, R. (2001). Physics and Creativity: Problem Solving and Learning Contexts. Industry and Higher Education, 15(6) 431-439. doi: http://dx.doi.org/10.5367/000000001101295939

Becerra, G. Y. \& Gallego, D. (2010). La gestión del conocimiento pedagógico en la Universidad Bolivariana de Venezuela, Táchira. Acción Pedagógica, 19, 38 - 51. Recuperado de http:// www.saber.ula.ve/bitstream/123456789/31925/1/articulo4.pdf

Canals, A. (2003). Gestión del conocimiento. Barcelona: Gestión 2000.

Correa, U. G., Jiménez, R. S. \& Segura, H. (2008). Diseño de un modelo de gestión del conocimiento para la Escuela Interamericana de Bibliotecología. Revista Interamericana de Bibliotecología, 31(1), 85-108. Recuperado de http://eprints.rclis.org/12325/1/ ARTICUL04.pdf

Davenport, T. \& Prusak, L. (2001). Conocimiento en Acción. Cómo las organizaciones manejan lo que saben. Buenos Aires: Editorial Prentice May

Drew, S., \& Smith, P. (1995). The Learning Organization: Change Proofing and Strategy. The Learning Organization, 2(l), 4-14. doi:10.1108/09696479510075598

Duval, R. (1993). Registres de répresentation sémiotique et functionement cognitif de la pensée (Registers of semiotic representations and cognitive functioning of thinking), Annales de Didactique et de Sciences Cognitives, 5, 35-75. doi:10.1007/ s10649-006-0400-z

Martin Fernández, E., (2001).Gestión de Instituciones Educativas Inteligentes. Manual para gestionar cualquier tipo de organización. España: MacGraw-Hill, Interamericana de España S.A.
Nieminen, M. (2001). Managing Human-centered Design Artifacts in Distributed Development Environment with Knowledge Storage. En M. J. Smith, G. Salvendy, D. Harris, \& R. J. Koubek (Eds.). Usability Evaluation and Interface Design: Cognitive Engineering, Intelligent Agents and Virtual Reality. Volumen 1 (pp. 988-992). Mahwah, New Jersey: Lawrence Erlbaum Associates.

Nonaka, I., \& Takeuchi, H. (1995).The Knowledge Creation Company: How Japanese Companies Create the Dynamics of Innovation. New York: Oxford University Press.

Oliveros, R. (2012). Gerencia del conocimiento a través de las TIC en la municipalización de los Programas Nacionales de Formación (PNF) en las Universidades e Institutos Universitarios. Revista arbitrada del Centro de Investigación y Estudios Gerenciales A.C., 3(2), 54-66.

Ongallo, C. (2003). Gestión del conocimiento. Las habilidades directivas. Madrid: Díaz de Santos.

Peluffo A. M. B. \& Catalán, C. E. (2002). Introducción a la gestión del conocimiento y su aplicación al sector público. Santiago de Chile: Instituto Latinoamericano y del Caribe de Planificación Económica y Social - ILPES. Recuperado de http://archivo. cepal.org/pdfs/2002/S2002617.pdf

Pérez, J. (2002). ¿Qué aporta la gestión del conocimiento a la formación? Ponencia presentada XVIII Simposio Internacional de Computación en Educación, SOMECE (Eds.), Zacatecas, México. Recuperado de http://www.somece.org.mx/memorias/2002.

Rodríguez, A.; Araujo, A. \& Urrutia, J. (2001). La gestión del conocimiento científico técnico en la universidad: un caso y un proyecto. Cuadernos de gestión, 1(1), 13-30. Recupera-do de http://www. ehu.es/cuadernosdegestion/documentos/111.pdf

Romero, A. (2005). Acerca de la Gestión del Conocimiento y la Innovación Tecnológica en el Proyecto BID/FONACIT II. Revista Digital CENIAP HOY, 8, 1-4. Recuperado de http://sian.inia.gob.ve/ repositorio/revistas_tec/ceniaphoy/articulos/n8/arti/romero_a/ romero_a.htm

(c) Los autores. Este artículo es publicado por la Revista Digital de Investigación en Docencia Universitaria del Área de Institutional Research and Effectiveness de la Dirección de Aseguramiento de la Calidad, Universidad Peruana de Ciencias Aplicadas. Este es un artículo de acceso abierto, distribuido bajo los términos de la LicenciaCreativeCommons Atribución-Compartirlgual 4.0 Internacional.( http://creativecommons.org/licenses/by-sa/4.0/), que permite el uso no comercial, distribución y reproducción en cualquier medio, siempre que la obra original sea debidamente citada. 